\title{
LOS MODELOS DE EXPLOTACIÓN PETROLERA DE ECUADOR Y DE COLOMBIA: UN ANÁLISIS DESDE EL EXTRACTIVISMO Y EL NEOEXTRACTIVISMO (SEGUNDA PARTE)
}

\author{
Por: Luis Hernando Portillo Riascos ${ }^{1}$
}

\section{RESUMEN}

En la primera parte de este trabajo se describieron los elementos conceptuales alrededor de los dos tipos o estilos de extractivismo. A la vez, se realizó una exposición de los principales cambios en el marco regulatorio del sector petrolero en los dos países analizados: Ecuador y Colombia. La segunda parte, que aquí se presenta, tiene como objetivo, hacer una revisión empírica de los dos modelos de explotación petrolera.

En el análisis cuantitativo se encontró diferencias importantes en la evolución del sector petrolero de los dos países. En el caso ecuatoriano, el Estado fue capaz de desarrollar una política activa centrada en capturar una mayor parte de la renta petrolera. En Colombia, por su parte, se estructuró un marco regulatorio orientado a la atracción de capital, que en un contexto de subida de los precios, hizo posible un incremento significativo de la producción petrolera. Ahora bien, haciendo la comparación de los referentes conceptuales en torno a los dos tipos de extractivismo y la evolución de la dinámica productiva y fiscal, las divergencias ya no son tan evidentes.

Palabras clave: extractivismo, neoextractivismo, modelos de explotación petrolera, Colombia, Ecuador.

Clasificación JEL: O13, Q33, Q48.

1. Máster en Economía Internacional y Desarrollo de la Universidad Complutense de Madrid. Economista y Especialista en Finanzas de la Universidad de Nariño. Docente tiempo completo adscrito al Programa de Economía de la Universidad de Nariño. Integrante Grupo de Investigación CES - Universidad de Nariño. E-mail: her182000@yahoo.es. Agradezco los comentarios y observaciones de María José Paz Antolín. 


\title{
MODELS OF OIL EXPLOITATION OF ECUADOR AND COLOMBIA: AN ANALYSIS FROM THE EXTRACTIVISM AND NEOEXTRACTIVISMO (SECOND PART)
}

\author{
By: Luis Hernando Portillo Riascos
}

\begin{abstract}
In the first part of this work described the conceptual elements around the two types or styles of extractivism. At the same time, gave a presentation of the main changes in the regulatory framework of the oil sector in two countries analyzed: Ecuador and Colombia. The second part, which here appears, has like target, make an empirical review of two models of oil exploitation.

In the quantitative analysis was found important differences in the evolution of the oil sector of the two countries. In the Ecuadorian case, the State was capable to develop an active policy focused on capturing a greater share of the oil income. In Colombia, for his part, was structured a regulatory framework aimed at attracting of capital, that in a context of increase of the prices, made possible a significant increase of the oil production. However, doing the comparison of the conceptual referents concerning to the two types of extractivism and the evolution of the productive and fiscal dynamic the divergence aren't so obvious.
\end{abstract}

Keywords: extractivism, neoextractivismo, oil exploitation models, Colombia, Ecuador.

JEL classification: O13, Q33, Q48. 


\title{
OS MODELOS DE EXPLORAÇÃO PETROLEIRA DO EQUADOR E DA COLOMBIA: UMA ANÁLISE DESDE O EXTRATIVISMO E O NEOEXTRATIVISMO (SEGUNDA PARTE)
}

\author{
Por: Luis Hernando Portillo Riascos
}

\begin{abstract}
RESUMO
Na primeira parte deste trabalho descreveram-se os conceitos ao redor dos dois tipos ou estilos de extrativismo. Ao mesmo tempo fez-se uma exposição das principais mudanças no marco regulamentar do setor petroleiro nos principais países analisados: Equador e Colombia. A segunda parte, que aqui se apresenta, tem como objetivo, fazer uma revisão empírica dos modelos de exploração petroleira.

$\mathrm{Na}$ análise quantitativa encontraram-se diferenças importantes na evolução do setor petroleiro dos dois países. No caso equatoriano, o Estado foi capaz de desenvolver uma política ativa centrada na captura de uma maior percentagem petroleira. Por sua parte, na Colombia, estruturou-se um marco regulador orientado a atrair capital, que num contexto de subida dos preços, fez possível um aumento significativo da produção petroleira. Ora pois, fazendo a comparação dos referentes conceitos à volta dos dois tipos extrativismo e a evolução da dinâmica produtiva e fiscal, as divergências já não são tão evidentes.
\end{abstract}

Palavras Chave: extrativismo, neoextrativismo, modelos de exploração petroleira, Colombia, Ecuador.

Classificação JEL: O13, Q33, Q48. 


\section{INTRODUCCIÓN}

En la primera parte de esta investigación se realizó un acercamiento preliminar a las diferencias entre los dos tipos o estilos de extractivismo, el clásico o convencional y el neoextractivismo. Para ello se analizaron los aportes de la literatura sobre este tema, y posteriormente se hizo una revisión de los cambios más importantes en el marco regulatorio del sector petrolero en los dos países analizados: Colombia y Ecuador. Esta segunda parte tiene como objetivo, describir las principales características de los modelos petroleros de estos dos países a partir de información empírica.

Se analiza el sector petrolero por varias consideraciones: en primer lugar, por la importancia que adquiere en las economías de los dos países seleccionados; en segundo lugar, porque se hace posible la comparación dado que se pueden homogeneizar criterios para el análisis empírico; en tercer lugar, por la funcionalidad para el contraste entre los dos tipos de extractivismo; y por último, por las implicaciones que tendría en la estrategia de desarrollo de cada uno de los países, aunque esta última cuestión excede los límites de este estudio.

El periodo de análisis comprende desde el año 2000 hasta 2012. Las razones por las cuales se escoge este lapso de tiempo son tres: en primer lugar, el planteamiento del neoextractivismo se realiza en el marco de la llegada de gobiernos progresistas en AL, lo cual solamente sucede hacia mediados de la década de los $2000^{2}$; en segundo lugar, los cambios en el marco regulatorio, en el caso colombiano, se empiezan a realizar desde el año 2002; y por último, el denominado "boom de las materias primas" se ubica particularmente dentro de este periodo, señalando su inicio entre los años 2003 y 2004.

Para el análisis de los modelos de explotación petrolera se abordaron dos dimensiones: la productiva y la fiscal. En la primera se hace una revisión de la evolución del sector petrolero en cada uno de los países estudiados. En la segunda, se describe la importancia del petróleo en las cuentas públicas, donde, por una parte, se analiza la capacidad del Estado para captar la renta petrolera, y por otra, se evalúa el uso que se le ha dado a la misma. La idea es aportar información que permita ahondar en las diferencias entre los dos tipos o estilos de extractivismo.

\section{DIMENSIÓN PRODUCTIVA}

En esta parte del estudio se hará referencia a las características generales de la cadena del petróleo en los dos países. La información será presentada siguiendo las fases que implica el proceso de explotación petrolera.

\section{Reservas}

La información obtenida para el periodo de análisis muestra un incremento de las actividades de prospección en los dos países. Sin embargo, esta dinámica no solamente se explica por los cambios recientes en el marco regulatorio. Aun así,

2. En el caso de Ecuador sería a partir de 2007 con la llegada de Rafael Correa a la presidencia; no obstante, Gudynas (2013) plantea el uso del término "neoextractivismo" desde 2008. 
ya se puede vislumbrar los efectos generados por la nueva configuración de sus modelos petroleros.

El Cuadro 1 muestra que hay una diferencia significativa en cuanto al volumen de reservas de los dos países, la cual ha tendido a crecer en los últimos años. Esta dinámica obedece, inicialmente, a que en el caso ecuatoriano la reposición de reservas es muy superior a su explotación, entre tanto, en Colombia, lo que se viene haciendo es reemplazarlas a un ritmo similar al de la producción. Para explicar esta situación se debe tener en cuenta que el stock de reservas depende de factores tales como: expectativas sobre los precios del petróleo, inversiones en exploración, intensidad de la explotación, condiciones técnicas que hagan posible dicha producción, potencial geológico del país etc.

Cuadro 1. Reservas de petróleo (miles de millones de barriles)

\begin{tabular}{|l|c|c|c|c|c|c|c|c|c|c|c|c|c|}
\hline \multicolumn{1}{|c|}{ Años } & 2000 & 2001 & 2002 & 2003 & $\mathbf{2 0 0 4}$ & $\mathbf{2 0 0 5}$ & $\mathbf{2 0 0 6}$ & $\mathbf{2 0 0 7}$ & $\mathbf{2 0 0 8}$ & $\mathbf{2 0 0 9}$ & $\mathbf{2 0 1 0}$ & $\mathbf{2 0 1 1}$ & $\mathbf{2 0 1 2}$ \\
\hline Colombia & 2.0 & 1.8 & 1.6 & 1.5 & 1.5 & 1.5 & 1.5 & 1.5 & 1.4 & 1.4 & 1.9 & 2.0 & 2.2 \\
\hline Ecuador & 4.6 & 4.6 & 5.1 & 5.1 & 5.1 & 4.9 & 4.5 & 4.0 & 6.5 & 6.3 & 6.2 & 7.2 & 8.2 \\
\hline
\end{tabular}

Fuente: British Petroleum (BP) - Statiscal Review of World Energy - 2013

En el caso colombiano, uno de los aspectos preocupantes es la ratio reservasproducción, que para el año 2012 alcanzaba, solamente, 6.89 años (UPME, 2013: 105). Si las reservas no crecen, en pocos años se tendría que importar petróleo. Este hecho explicaría la política agresiva del gobierno nacional para atraer inversión, tal y como se pudo ver en el apartado del marco regulatorio (Portillo, 2014). En el caso de Ecuador, la situación es muy diferente; con el nivel alcanzado en 2012, y manteniéndose estables las demás condiciones, sus reservas alcanzarían para 44 años. De ahí que los dos países se enfrentan a condiciones distintas, y su política petrolera, a priori, no tendría necesariamente que coincidir.

En Colombia, los grandes descubrimientos de petróleo se hicieron en los 80s y 90s en los yacimientos de Caño Limón, y luego en Cusiana y Cupiagua. De ahí en adelante, a pesar de que se han tenido resultados positivos, las magnitudes no han sido significativas. Si a esto se le une el incremento de la producción en la segunda parte de la década de los 2000, la situación se hace más compleja todavía.

No obstante, hay un incremento sustancial de las actividades de prospección. Entre 2004 y 2012, se levantaron 152.820 kilómetros de sísmica, con un promedio de 16.580 kilómetros por año. Asimismo, el número de pozos perforados (A-3 $\left.{ }^{3}\right)$ durante los últimos siete años, ascendieron a 611, que son comparables con los realizados durante las dos décadas anteriores. Cabe resaltar que dichas labores fueron realizadas, en su gran mayoría, por empresas privadas ${ }^{4}$. La presencia de Ecopetrol en esta clase de actividades ha sido muy reducida, tanto en las labores

3. Clasificación utilizada en el lenguaje petrolero, y corresponde al primer pozo que se perfora en un área geológicamente inexplorada (UPME, 2013: 102)

4. Ecopetrol perforó durante los últimos seis años, de manera directa, 19 pozos; con asociados, 122; y en convenio con la ANH, 35. 
directas como en los convenios con la ANH y con los asociados ${ }^{5}$. La inversión realizada por esta empresa en actividades de exploración durante el periodo 2004 - 2011 fue de US\$ 2.635 millones $^{6}$.

En el caso de Ecuador, el ritmo de crecimiento de las reservas petroleras es alta, como se señaló anteriormente, con una media anual del $15.5 \%$ entre 2007 y 2012. Dentro de los factores que explican dicho comportamiento se encuentran, por una parte, las reformas en el marco regulatorio realizadas durante la década de los noventas e inicio del nuevo siglo, donde la característica más importante es la apuesta por una política de atracción de inversión extranjera (Campodónico, 2004 y 2007); y por otra, el estancamiento de la producción que se presenta en los últimos años.

Al igual que en Colombia, las labores de prospección también se han incrementado. Entre los años 2007 al 2012 se perforaron 1053 pozos, entre exploratorios (38), de desarrollo (989) y, de avanzada (26) ${ }^{7}$. Es importante anotar que las compañías privadas redujeron la participación en este tipo de actividades. Entre 2007 y 2012, los pozos perforados por empresas públicas fueron 766 (73\%), entretanto, las privadas realizaron 287 (23\%). Aquí se puede ver un cambio drástico respecto al año 2007 donde se perforaron 109 pozos, de los cuales, 77 fueron realizados por empresas privadas $(70.64 \%)^{8}$. De esta manera, el efecto de los cambios en el marco regulatorio es muy claro. Dichas medidas le van permitiendo al Estado, tener un mayor control sobre la actividad petrolera, lo cual es consecuente con los planteamientos neoextractivistas.

\section{Producción de crudo}

La producción de petróleo presenta distintas evoluciones en los países de estudio. En el caso colombiano, hay un incremento notable, fundamentalmente durante los últimos seis años (ver Cuadro 2). La tasa media (anual) de crecimiento durante este periodo fue del $12 \%$, con una variación acumulada del $77 \%$. La situación de Ecuador es distinta, mientras en el periodo 2000-2006 la producción venía creciendo, a partir de 2007, empieza a descender, y permanece estancada hasta el 2012.

Por otra parte, mientras en Colombia la participación de empresas públicas no se ha modificado sustancialmente, aunque permanece alta, en Ecuador, sucede lo contrario. En el año 2006 las empresas estatales producían el 46\% del petróleo, en 2012, dicha participación pasó a ser del 73\%. La modificación de los contratos petroleros, aunado a la declaración de caducidad de los contratos de Occidental

5. Entre 2009 y 2012, Ecopetrol no firmó ningún contrato directo. Sí se realizaron a través de asociados y en convenio, aunque la participación global es mínima.

6 Datos página web de Ecopetrol (Consultado 3 de marzo de 2014) y SIPG. http://www.ecopetrol. com.co /contenido.aspx?catID $=44 \&$ conID $=76465$. En cuanto a las inversiones en exploración realizadas por empresas privadas, no se encontraron datos disponibles.

7. Los pozos exploratorios tienen como propósito hacer nuevos descubrimientos. Los de desarrollo, representan el primer pozo que se perfora en un yacimiento para encontrar petróleo. Los de avanzada sirven para delimitar la estructura del yacimiento.

8. Datos Ministerio de Minas y Petróleos. No se encontraron datos disponibles sobre inversiones en exploración realizadas por empresas privadas. 
(OXY) en el año 2006 y posteriormente el de la empresa Perenco en 2010, cuyas labores fueron asumidas por empresas públicas, permitieron que se pueda dar esta situación. En el caso colombiano no se observan cambios importantes puesto que Ecopetrol conserva la gran mayoría de los contratos de asociación que se habían firmado hasta 2003. En la medida que se incorporen más reservas, fruto de los nuevos contratos (E\&P y TEA), la situación puede cambiar sustancialmente.

Cuadro 2. Producción de petróleo (crudo). Periodo 2000-2012

\begin{tabular}{|c|c|c|c|c|c|c|}
\hline \multirow[t]{2}{*}{ Años } & \multicolumn{2}{|c|}{$\begin{array}{c}\text { Producción diaria de crudo } \\
\text { (Miles de barriles) }\end{array}$} & $\begin{array}{c}\text { Empres as } \\
\text { Públicas (\%)* }\end{array}$ & $\begin{array}{c}\text { Empres as } \\
\text { Privadas (\%) }\end{array}$ & $\begin{array}{c}\text { Empres as } \\
\text { Públicas }(\%)^{* *}\end{array}$ & $\begin{array}{c}\text { Empresas } \\
\text { Privadas }(\%)^{* * * *}\end{array}$ \\
\hline & Colombia & Ecuador & \multicolumn{2}{|c|}{ Colombia } & \multicolumn{2}{|c|}{ Ecuador } \\
\hline 2000 & 687 & 403 & 66 & 34 & 58 & 42 \\
\hline 2001 & 604 & 410 & 58 & 42 & 56 & 44 \\
\hline 2002 & 578 & 394 & 57 & 43 & 56 & 44 \\
\hline 2003 & 541 & 420 & 57 & 43 & 49 & 51 \\
\hline 2004 & 528 & 528 & 58 & 42 & 37 & 63 \\
\hline 2005 & 526 & 534 & 59 & 41 & 37 & 63 \\
\hline 2006 & 529 & 538 & 60 & 40 & 46 & 54 \\
\hline 2007 & 531 & 513 & 60 & 40 & 51 & 49 \\
\hline 2008 & 588 & 507 & 60 & 40 & 53 & 47 \\
\hline 2009 & 671 & 488 & 61 & 39 & 58 & 42 \\
\hline 2010 & 786 & 488 & 61 & 39 & 62 & 38 \\
\hline 2011 & 915 & 501 & 62 & 38 & 72 & 28 \\
\hline 2012 & 944 & 505 & 63 & 37 & 73 & 27 \\
\hline
\end{tabular}

Fuente: elaboración propia con datos del Banco Central del Ecuador, Sistema de Información de Petróleo y Gas (SIPG) y Ecopetrol

* EP Petroecuador, Petroamazonas y Rio Napo. Dentro de las empresas privadas, las más importantes serían: Repsol y Andes Petroleum.

** Se suma la producción de Ecopetrol realizada de forma directa y la que hace a través de contratos de asociación.

*** La empresa más importante por volumen de producción es Meta Petroleum Limited. Luego, hay un gran número de empresas, aunque con un tamaño mucho menor (Occidental de Colombia, Equion Energia LTD., Mansarovar Energy Colombia LTD., Cepcolsa, Gran Tierra Energy Colombia LTD, Petrobras Colombia Limited etc.)

De esta manera, los volúmenes de producción alcanzados durante los últimos años, muestran, por un lado, la apuesta realizada por cada uno de los países, y por otro, las limitaciones que puede tener su modelo de explotación. En el caso de Colombia, el objetivo central era incrementar la producción, algo que en el periodo analizado, se ha cumplido. En Ecuador, aun cuando se logra que la participación del Estado, a través de las empresas públicas, pase a tener un peso elevado en la producción petrolera, no muestra signos de capacidad de incrementar la producción más allá de la que se ha tenido en los últimos años. Se podría decir que, hasta el momento, lo único que ha cambiado es la propiedad del recurso (petróleo) y quien lo extrae, más no se puede observar una transformación y una política orientada a incrementar la producción.

La dinámica de la producción petrolera en Ecuador es consecuente con los planteamientos neoextractivistas. Por otro lado, las modificaciones en la regulación no resultan suficientes y se pueden ver las grandes barreras a las que se enfrenta. No obstante, el fenómeno descrito contradice algunos de las posiciones planteadas por los críticos del neoextractivismo, en el sentido de que se sigue apostando por 
atraer a las transnacionales para incrementar la producción. Los datos muestran un crecimiento en participación de las empresas públicas. Empero, la producción no ha crecido, a diferencia de lo que ha sucedido en el caso colombiano.

\section{Inversión extranjera en el sector petrolero}

Los cambios introducidos en el marco regulatorio tienen repercusiones en el rol que entra a asumir la Inversión Extranjera Directa (IED) en el sector petrolero. Uno de los hechos más representativos es la dinámica que toma la producción de petróleo (crudo) en los dos países. En el periodo 2004-2006, Ecuador y Colombia tenían niveles de producción similares, sin embargo, a partir de 2007, mientras en el primer caso se presenta un estancamiento, en el segundo, hay un crecimiento notable. De forma paralela, la IED sufre un cambio muy marcado. Entretanto en Ecuador, hay una reducción y luego niveles de inversión relativamente bajos, en Colombia, pasa todo lo contrario, hay un crecimiento significativo. En el periodo 2007 - 2012 la IED en el sector petrolero realizada en Ecuador fue de US\$ 929 millones, mientras en Colombia el monto ascendió a US\$22.402 millones. De ahí que el rol que asume la IED en los dos países genera una diferencia importante en la trayectoria productiva durante los últimos años (ver Cuadro 3).

\section{Cuadro 3. Inversión Extranjera Directa en petróleo (millones de dólares)}

\begin{tabular}{|c|c|c|c|c|}
\hline \multirow{2}{*}{ Año } & \multicolumn{2}{|c|}{ IED sector petrole ro } & \multicolumn{2}{c|}{ IED en sector petrole ro como \% IED total } \\
\cline { 2 - 5 } & Ecuador * & Colombia** & Ecuador & Colombia \\
\hline 2000 & $(59)$ & -384 & 123 & -16 \\
\hline 2001 & 217 & 521 & 40 & 20 \\
\hline 2002 & 487 & 449 & 62 & 21 \\
\hline 2003 & 149 & 278 & 17 & 16 \\
\hline 2004 & 385 & 495 & 46 & 16 \\
\hline 2005 & 198 & 1.125 & 40 & 11 \\
\hline 2006 & $(117)$ & 1.995 & -43 & 30 \\
\hline 2007 & $(103)$ & 3.333 & -53 & 37 \\
\hline 2008 & 244 & 3.405 & 23 & 32 \\
\hline 2009 & 6 & 2.428 & 2 & 34 \\
\hline 2010 & 178 & 2.785 & 109 & 41 \\
\hline 2011 & 379 & 5.062 & 59 & 38 \\
\hline 2012 & 225 & 5.389 & 38 & 34 \\
\hline
\end{tabular}

Fuente: elaboración propia con datos del Banco Central del Ecuador y Banco de la República.

* Se toma la IED para minas y canteras (incluye petróleo y gas) puesto que no se discrimina más allá de las grandes ramas de la actividad económica. La participación de la minería es poco significativa. Los datos presentados están sujetos a revisión a partir del 2007 (Banco Central del Ecuador).

** Datos provisionales para los años 2003 y 2004, y preliminares para los años 2005 al 2012 (Banco de la República) 
Se resalta que el volumen de IED realizada en el sector petrolero resulta significativo respecto del total. En el caso de Colombia la situación es muy notoria, pudiéndose visualizar con mayor intensidad a partir de 2005. Así, en los últimos siete años, en promedio, de la IED total, el 35\% fue direccionado al sector petrolero. En el caso de Ecuador se deben introducir algunos matices: en los años 2006 y 2007, la desinversión bruta es superior a la IED; dicha situación se revierte, en parte, a partir de 2010; no obstante, los montos son relativamente bajos. De cualquier manera, la IED sigue concentrada en la actividad petrolera.

Así, se puede observar una dinámica divergente entre los dos países, que denota distintas estrategias, y por tanto modelos de explotación petrolera claramente diferenciados, donde el papel que entra a jugar la IED resulta fundamental para entenderlos. Esta situación es coherente si se tiene en cuenta las diferencias de los dos tipos o variantes de extractivismo.

\section{Producción de derivados}

Una de las paradojas que se cita en la literatura para criticar a los países que se especializan en la explotación de petróleo, es que, aun siendo los propietarios del recurso, muchas veces se ven obligados a importar derivados, dada su incapacidad para realizar el proceso de transformación. A la vez, quienes defienden la idea de la industrialización a partir de los recursos naturales ${ }^{\mathbf{9}}$, hacen hincapié en la necesidad de avanzar en la cadena de valor por esta vía.

En el Cuadro 4 se muestra el crecimiento en la producción de derivados y la relación entre la demanda y la oferta de este tipo de productos. Como se puede ver, la mayor característica es que, mientras Colombia tiene una capacidad productiva superior al consumo, en el caso de Ecuador, su producción no alcanza para abastecer la demanda interna.

En el caso ecuatoriano se muestran distintos elementos: primero, el consumo de derivados, en general, se incrementa de manera muy notable, superando la producción desde 2005, y acrecentando el desequilibrio en los últimos años; segundo, hay un crecimiento acelerado en el consumo de gasolina, que para el periodo 2007-2012 aumentó a una tasa media anual del 7.9\%; tercero, el crecimiento de las importaciones es bastante significativo (tasa media anual del 13.9\%); y por último, la participación de los productos derivados en el total de importaciones tiende a profundizarse, representado, entre 2006 y 2012, como media, el 20\% De esta manera, la tendencia no se ha modificado con el cambio de gobierno en 2007; por el contrario, se agudiza. Este elemento representa una clara restricción del modelo petrolero ecuatoriano, y más en un marco donde se intenta reducir el peso de las actividades extractivas.

El caso colombiano presenta algunas diferencias. En primer lugar, el consumo de gasolina disminuye (aumenta el de diesel); en segundo lugar, tanto el consumo global de derivados como la producción, crecen a ritmos lentos. De todas maneras, hay un excedente importante que permite al país ser exportador neto (hasta 2011).

9. Ver Pérez (2010) y Ramos (1999).

10. Los productos que más se importan son: diésel, nafta y Gas Licuado de Petróleo (GLP). 
No obstante, las importaciones se incrementan a un ritmo muy alto, particularmente en los últimos tres años. Esto hace que al finalizar el 2012, la participación de los combustibles y lubricantes en el total de importaciones llegue a representar el 9.7\%.

Así, mientras la producción de crudo creció a un ritmo importante, la de derivados presenta una situación distinta. No obstante, no se puede desconocer que es un país con cierta capacidad de refino, como se verá en adelante, y no meramente extractivista. De otro lado, las modificaciones en el marco regulatorio han tenido mayores efectos en la cuanto a la explotación de crudo, no así en el caso de los derivados.

\section{Cuadro 4. Producción y consumo de derivados del petróleo. Periodo 2000-2012}

\begin{tabular}{|c|c|c|c|c|c|c|c|c|c|c|}
\hline \multirow[t]{2}{*}{ Año } & \multicolumn{2}{|c|}{$\begin{array}{c}\text { Consumo de Gasolina } \\
\text { (cifras equivalentes en } \\
\text { barriles de petróleo) }\end{array}$} & \multicolumn{2}{|c|}{$\begin{array}{c}\begin{array}{c}\text { Consumo global derivados } \\
\text { de petróleo (barriles } \\
\text { diarios) }\end{array} \\
\end{array}$} & \multicolumn{2}{|c|}{$\begin{array}{l}\text { Producción derivados } \\
\text { (barriles diarios) }\end{array}$} & \multicolumn{2}{|c|}{$\begin{array}{l}\text { Importación de derivados } \\
\text { (Barriles diarios) }\end{array}$} & \multicolumn{2}{|c|}{$\begin{array}{c}\text { Participación derivados y } \\
\text { lubricantes en total de } \\
\text { importaciones }(\%)\end{array}$} \\
\hline & Colombia & Ecuador & Colombia & Ecuador & Colombia & Ecuador & Colombia & Ecuador & Colombia & Ecuador \\
\hline 2000 & 102.725 & 31.781 & 241.360 & 137.100 & 360.466 & 161.480 & 9.240 & 24.785 & 2,0 & 8,0 \\
\hline 2001 & 92.248 & 33.151 & 226.707 & 137.251 & 381.936 & 155.641 & 9.770 & 34.400 & 1,5 & 5,5 \\
\hline 2002 & 90.459 & 34.795 & 221.162 & 145.576 & 368.389 & 161.012 & 9.350 & 40.018 & 1,5 & 4,4 \\
\hline 2003 & 88.612 & 34.247 & 221.851 & 151.175 & 378.969 & 152.924 & 8.710 & 47.427 & 1,7 & 12,1 \\
\hline 2004 & 84.367 & 36.164 & 225.329 & 154.565 & 384.137 & 163.502 & 7.060 & 47.528 & 1,6 & 13,8 \\
\hline 2005 & 82.618 & 38.082 & 230.244 & 169.154 & 236.526 & 168.823 & 16.250 & 60.750 & 2,6 & 17,6 \\
\hline 2006 & 76.570 & 41.096 & 234.863 & 179.654 & 357.154 & 157.264 & 14.160 & 71.048 & 2,6 & 21,0 \\
\hline 2007 & 74.065 & 44.110 & 234.282 & 182.749 & 361.121 & 178.461 & 10.660 & 88.024 & 2,8 & 19,9 \\
\hline 2008 & 71.530 & 47.945 & 233.728 & 188.074 & 357.800 & 185.077 & 30.320 & 86.233 & 4,6 & 18,9 \\
\hline 2009 & 69.719 & 51.507 & 241.256 & 190.667 & 342.848 & 187.795 & 37.890 & 94.783 & 3,6 & 17,5 \\
\hline 2010 & 74.595 & 55.890 & 256.651 & 220.420 & 339.699 & 167.916 & 67.380 & 121.732 & 5,1 & 21,1 \\
\hline 2011 & 77.688 & 61.370 & 271.329 & 226.117 & 405.067 & 193.547 & 90.300 & 112.386 & 7,1 & 22,1 \\
\hline 2012 & 73.653 & 64.658 & 273.951 & 233.848 & 403.106 & 196.071 & 104.440 & 117.851 & 9,7 & 22,7 \\
\hline
\end{tabular}

\section{Capacidad de refino}

Las medidas tendientes a aumentar la producción de derivados, dados los problemas previamente señalados, tienen mucha más relevancia para Ecuador, principalmente por los desajustes en la balanza comercial, que al final terminan afectando las finanzas públicas (por la vía de los subsidios a los combustibles). De ahí que en los últimos años se pudo observar un crecimiento en la producción de gasolina, que entre 2002 y 2012, prácticamente se duplicó. Empero, en los demás productos, principalmente diésel, no se observa un aumento, por el contrario, tiende a disminuir. Cabe advertir que no es fácil, de un momento a otro, cambiar la matriz energética; uno de los principales escollos es la capacidad instalada existente, dado que no es suficiente para atender las necesidades de consumo doméstico.

Ecuador cuenta actualmente con 5 refinerías (propiedad estatal). Por el volumen de producción las más importantes son: Esmeraldas (57.12\%), Libertad (31.28\%) y Amazonas (9.59\%). Su capacidad de refinación global es de 175 mil barriles diarios (MBD) (Campodónico, 2007). Debido a las dificultades para atender la demanda interna, se han emprendido tareas para afrontar este grave problema. Desde 2007 se viene trabajando en el proyecto de construcción de la Refinería del Pacífico "Eloy Alfaro”, cuya capacidad prevista es de 300 MBD. En la etapa inicial se podrían 
refinar hasta $200 \mathrm{MBD}^{11}$. Debido a las consideraciones señaladas anteriormente, este proyecto ha sido calificado como estratégico para el país.

En el caso de Colombia, se cuenta con 5 refinerías, con una capacidad global de carga de $333 \mathrm{MBD}$. Las más importantes son: la de Barrancabermeja y la de Cartagena ${ }^{12}$. Actualmente se están desarrollando proyectos para la ampliación de la capacidad de refinación, que se incrementaría en $200 \mathrm{MBD}^{13}$. Las inversiones demandadas en estos proyectos habrían ascendido a US\$ 2400 millones de dólares entre 2007 y 2012 (UPME, 2013) ${ }^{14}$. El propósito de estas iniciativas es, por una parte, ampliar la capacidad de refino, y por otra, mejorar la calidad de los combustibles que se están vendiendo. Estos proyectos contribuirán a incrementar la oferta de derivados, en especial de diésel, dado que las importaciones, como ya se anotó, se vienen incrementando a un ritmo muy acelerado durante los últimos años.

A partir de los datos mostrados, se pudo observar que la capacidad de refino de Colombia es mucho mayor que la de Ecuador, lo cual deja ver la importancia de las condiciones previas. Por otro lado, señalar a Colombia como un extractivista clásico, tal y como lo hacía Gudynas (2011c y 2012), no sería del todo acertado.

\section{Exportaciones}

La importancia que tiene el petróleo en los dos países, a pesar de las diferencias, se reflejará en varios indicadores, uno de ellos, la participación en las exportaciones totales. Como se puede ver en el Cuadro 5, la característica común es que hay una tendencia a que las exportaciones de hidrocarburos tengan un mayor peso en las exportaciones totales. No obstante, hay diferencias importantes. Mientras en Ecuador dicha tendencia se presenta, a pesar de que la oferta de exportación disminuye, en el caso de Colombia, la explicación se encuentra en que el aumento de la producción, se refleja, casi en su totalidad, en un incremento de las exportaciones. Así pues, mientras en el año 2006, Ecuador exportaba el 70\% de la producción, dicho porcentaje se había reducido al 61\% en 2012. En el caso de Colombia, dichos porcentajes son del $44 \%$ y $66 \%$ respectivamente.

En Ecuador, un hecho que no permite que se incremente la oferta de exportación, se encuentra en que, a la vez que no creció la producción, el consumo lo hizo a una tasa muy acelerada. En el periodo 2000 - 2012, el consumo (petróleo en todos los usos) creció a una tasa media anual del 4.6\%, entre tanto, la producción solamente lo hizo al 1.8\%. Si se toma como referencia el periodo $2007-2012$,

11. El avance global del proyecto es del $11.7 \%$ y de la construcción, $5.9 \%$. El proyecto se desarrolla bajo una alianza estratégica entre Petroecuador y PDVSA. Para impulsar el proyecto, se firma en 2013 un acuerdo de entendimiento entre el Estado ecuatoriano, la empresa petrolera estatal China CNPC y el Banco Industrial y Comercial (ICBC) del mismo país. (Consulta realizada el 13 de febrero de 2015. http://www.rdp.ec).

12. Por estructura de propiedad, se está hablando de un monopolio estatal cuyos activos pertenecen a Ecopetrol. Solamente existe una empresa privada que es la Refinería de Hidrocasanare, pero su capacidad de carga es reducida (10 MDB).

13. Las inversiones son realizadas por Ecopetrol.

14. Según Ecopetrol (2013), los avances alcanzados al finalizar el año 2012 fueron: 74\%, en la ampliación de la Refinería de Cartagena y, 14\%, en modernización de la Refinería de Barrancabermeja. Los datos oficiales sobre los costos definitivos, no se han publicado. 
las tasas serían del 5.1\% y 4.6\%. En Colombia, la producción de petróleo creció a una tasa media anual del 2.5\% (entre 2000 y 2012) y el consumo lo hizo al 1.1\%. Partiendo del año 2007 los porcentajes serían del 12.2\% y 3.2\% respectivamente.

Por otra parte, la dinámica observada en los dos países da cuenta de la importancia de los sectores extractivos, y en concreto, del petróleo. Y no solamente por su peso en las exportaciones totales, que de hecho, es muy elevado. El rasgo más importante es la gran participación que adquiere una actividad en la cual, la mayor parte del producto (crudo) se exporta sin procesar. De ahí se deriva la calificación de extractivistas, lo cual alimenta la crítica hacia las estrategias de estos países en la medida en que no se observan grandes cambios en su patrón de inserción externa.

Cuadro 5. Exportaciones de petróleo (crudo). Periodo 2000 - 2012.

\begin{tabular}{|c|c|c|c|c|c|c|c|c|}
\hline \multirow[t]{2}{*}{ Año } & \multicolumn{2}{|c|}{$\begin{array}{l}\text { Producción (miles de } \\
\text { barriles diarios) }\end{array}$} & \multicolumn{2}{|c|}{$\begin{array}{c}\text { Exportaciones (miles de } \\
\text { barriles diarios) }\end{array}$} & \multicolumn{2}{|c|}{$\begin{array}{l}\text { Producción destina a } \\
\text { exportar }(\%)\end{array}$} & \multicolumn{2}{|c|}{\begin{tabular}{|c|}
$\begin{array}{c}\text { Exportaciones de petróleo } \\
\text { (crudo) como \% del total de } \\
\text { exportaciones }\end{array}$ \\
\end{tabular}} \\
\hline & Colombia & Ecuador & Colombia & Ecuador & Colombia & Ecuador & Ecuador & Colombia \\
\hline 2000 & 687 & 401 & 384 & 236 & 56 & 59 & 43 & 30 \\
\hline 2001 & 604 & 407 & 284 & 246 & 47 & 60 & 31 & 21 \\
\hline 2002 & 577 & 394 & 292 & 231 & 51 & 59 & 37 & 21 \\
\hline 2003 & 540 & 421 & 230 & 253 & 43 & 60 & 38 & 19 \\
\hline 2004 & 528 & 527 & 219 & 355 & 41 & 67 & 50 & 17 \\
\hline 2005 & 526 & 532 & 221 & 361 & 42 & 68 & 54 & 18 \\
\hline 2006 & 527 & 536 & 219 & 374 & 42 & 70 & 55 & 18 \\
\hline 2007 & 531 & 511 & 243 & 339 & 46 & 66 & 52 & 18 \\
\hline 2008 & 587 & 506 & 275 & 349 & 47 & 69 & 56 & 24 \\
\hline 2009 & 670 & 486 & 351 & 328 & 52 & 67 & 46 & 25 \\
\hline 2010 & 786 & 485 & 444 & 336 & 56 & 69 & 51 & 33 \\
\hline 2011 & 915 & 500 & 598 & 289 & 65 & 58 & 53 & 41 \\
\hline 2012 & 944 & 505 & 623 & 308 & 66 & 61 & 54 & 44 \\
\hline
\end{tabular}

Fuente: elaboración propia con datos del Banco Central del Ecuador, Sistema de Información de Petróleo y Gas (SIPG) y British Petroleum(BP) - Statistical Review of World Energy (2013).

Para Ecuador, en el año 2011, no se incluyen 15.7 millones de barriles correspondiente a la Secretaría de Hidrocarburos por pago a las Cías. Privadas por concepto de tarifa por prestación de servicios (Nota Banco Central del Ecuador). La misma situación se presentaría en el año 2012. Si se toman estas cantidades la oferta de exportación crecería, llegando al nivel alcanzado en 2006 (70\%).

En cuanto a las exportaciones de derivados, éstas no tienen una participación muy alta dentro del total, y además, a pesar de las oscilaciones, la estructura no se modifica sustancialmente durante este periodo. Además, como se puede ver en el Cuadro 6, hay un importante efecto de los precios, ya que si se analiza el comportamiento de las cantidades, las tasas de crecimiento son totalmente diferentes. Empero, mientras en Colombia el crecimiento está dado tanto en volúmenes como en términos nominales, en Ecuador, hay una tendencia a la disminución de las exportaciones de derivados ${ }^{\mathbf{1 5}}$. Lo más preocupante para Ecuador es el deterioro

15. En el periodo 2000-2012 el volumen de exportaciones disminuyó un 36\%. Si se toma como punto de referencia el año 2007, la caída es del 34\%. En el caso de Colombia, entre 2000 y 2012 , el volumen de exportaciones de derivados aumentó un $22 \%$. 
del saldo en la balanza comercial de derivados, fenómeno que se profundiza a partir de 2005, pero continúa con un ritmo acelerado bajo el gobierno de Rafael Correa. En el año 2012, el déficit llega a representar el 5.2\% del PIB, algo que va a tener repercusiones directas en el presupuesto del gobierno, tal y como se señaló anteriormente. En el caso de Colombia, a pesar que en casi todos los años tiene un superávit, en 2012, el saldo pasa a ser deficitario.

Cuadro 6. Exportación de derivados y saldo comercial. Periodo 2000 - 2012

\begin{tabular}{|c|c|c|c|c|c|c|c|c|}
\hline \multirow[b]{2}{*}{ Años } & \multicolumn{4}{|c|}{ Colombia } & \multicolumn{4}{|c|}{ Ecuador } \\
\hline & $\begin{array}{c}\% \\
\text { exportaciones } \\
\text { totales }\end{array}$ & $\begin{array}{l}\text { miles de } \\
\text { US\$ }\end{array}$ & $\begin{array}{c}\text { Volumen } \\
\text { (miles de } \\
\text { barriles día } \\
\text { calendario) }\end{array}$ & \begin{tabular}{|c|} 
Balanza \\
comercial \\
derivados \\
(miles de US\$) \\
\end{tabular} & $\begin{array}{c}\% \\
\text { exportaciones } \\
\text { totales }\end{array}$ & $\begin{array}{c}\text { miles de } \\
\text { US\$ }\end{array}$ & \begin{tabular}{|c|} 
Volumen \\
(miles de \\
barriles día \\
calendario) \\
\end{tabular} & \begin{tabular}{|c|} 
Balanza \\
comercial \\
derivados \\
(miles de US\$) \\
\end{tabular} \\
\hline 2000 & 5,9 & 777.620 & 79,1 & 589.856 & 6,1 & 297.795 & 43,3 & (409) \\
\hline 2001 & 5,7 & 706.588 & 87,2 & 573.085 & 3,2 & 176.723 & 39,3 & (72.861) \\
\hline 2002 & 6,0 & 712.881 & 81,5 & 552.025 & 4,1 & 215.226 & 36,3 & (17.183) \\
\hline 2003 & 7,1 & 936.180 & 88,5 & 736.965 & 3,6 & 232.286 & 31,9 & (313.731) \\
\hline 2004 & 7,9 & 1.322 .271 & 101,5 & 1.109 .932 & 4,2 & 333.942 & 36,3 & (252.188) \\
\hline 2005 & 7,9 & 1.684.167 & 91,4 & 1.265 .395 & 4,5 & 470.596 & 35,1 & (1.104.830) \\
\hline 2006 & 7,8 & 1.895 .729 & 94,3 & 1.409 .178 & 4,6 & 599.874 & 37,3 & $(1.640 .107)$ \\
\hline 2007 & 6,5 & 1.942 .499 & 53,0 & 1.398 .638 & 5,7 & 846.094 & 41,5 & (1.666.107) \\
\hline 2008 & 8,5 & 3.213 .156 & 84,7 & 1.656 .237 & 5,9 & 1.145 .446 & 42,2 & $(2.179 .262)$ \\
\hline 2009 & 6,4 & 2.101 .002 & 82,3 & 902.574 & 4,7 & 675.075 & 33,8 & (1.555.576) \\
\hline 2010 & 8,6 & 3.405 .448 & 93,6 & 1.337 .588 & 3,9 & 712.706 & 28,1 & $(3.212 .302)$ \\
\hline 2011 & 9,1 & 5.151 .950 & 101,9 & 1.305 .616 & 4,8 & 1.102 .250 & 31,6 & (3.878.244) \\
\hline 2012 & 8,7 & 5.229 .923 & 96,6 & (484.415) & 4,1 & 1.013 .857 & 27,5 & (4.415.609) \\
\hline
\end{tabular}

Fuente: elaboración propia con datos del Sistema de Información de Petróleo y Gas (SIPG), Banco Central del Ecuador y DANE.

Los aspectos más resaltables de la primera dimensión analizada serían los siguientes: en primer lugar, la dinámica de la producción petrolera es distinta en los dos países, mientras en Colombia hay un crecimiento notable de la producción, en Ecuador, por el contrario, se estanca; en segundo lugar, hay un incremento de la participación del petróleo en la estructura de exportaciones, fundamentalmente de crudo, con lo cual, se refuerza el estilo extractivista; en tercer lugar, a pesar de que los dos países muestran cierta capacidad de refino, hay un desajuste entre el crecimiento de la producción interna y la demanda doméstica de derivados, y por último, los cambios en los marcos regulatorios en los dos países han tenido mucho más impacto en la producción de crudo que en la de derivados, no obstante, las condiciones previas y fenómenos externos como la subida en los precios del petróleo también han jugado un importante papel.

La orientación exportadora (crudo) permite entender los alcances y limitaciones de estos modelos, algo que el marco regulatorio no logra abordar. No obstante, aunque se muestran características propias del extractivismo, la importancia que adquiere el Estado, en el caso de Ecuador, muestra un cambio importante en su modelo de explotación petrolera. 


\section{DIMENSIÓN FISCAL}

Tal y como se planteó inicialmente, la idea es comprender cómo el Estado, a través de los instrumentos de política fiscal, participa en la renta petrolera y hace uso de la misma. Como se ha venido señalando a lo largo de esta investigación, este es un tema fundamental a la hora de entender la bondad de un modelo de explotación de recursos naturales (RRNN), y en particular, del petróleo.

\section{Ingresos}

Teniendo presente el grado de importancia que adquiere la actividad petrolera dentro del PIB y particularmente en las exportaciones de los dos países, lo que se espera es que la contribución al erario siga dicha trayectoria. En el Cuadro 7 se presentan los ingresos del Sector Público Nivel Central (SPNC) a partir de sus tres componentes principales: los impuestos directos (renta), los impuestos indirectos (Iva) y, los ingresos petroleros ${ }^{16}$.

Lo primero que se puede destacar es que, en general, la presión fiscal es baja en los dos países, alcanzando niveles cercanos al 20\% del PIB. No obstante, en Ecuador, fundamentalmente desde 2007, hay un mayor esfuerzo por incrementar la captación de rentas, en especial las petroleras, que de hecho son las más significativas. En Colombia, aunque el recaudo también se incrementa, no se generan modificaciones sustanciales en el conjunto de la estructura fiscal. Una de las explicaciones viene dada por el peso que tienen las rentas petroleras en el total del recaudo, donde, no es tan relevante para el caso de Colombia como si lo es para Ecuador.

Como ya se advertía en la revisión de literatura, la bonanza en los precios del petróleo da lugar a que los ingresos fiscales tiendan a concentrarse en las rentas derivadas de esta actividad (Portillo, 2014). La información aportada da luces que ratifican la presencia de este fenómeno. Así por ejemplo, mientras en 2003, los ingresos petroleros aportaban el 7.1\% de los ingresos del SPNC de Colombia, en 2012, dicho aporte había subido al 17\% (ver Cuadro 7). En el caso de Ecuador la participación sería del $32.7 \%$ y 31.2\% respectivamente. Por otra parte, mientras en Colombia el crecimiento es mucho más acelerado a pesar de que la base de partida es inferior, en Ecuador, dicho fenómeno es más visible desde el año 2007.

Ahora bien, a pesar de que la tendencia es la misma en los dos países, la explicación que se le puede dar es diferente. En Colombia, el mayor recaudo viene dado por un incremento importante de la producción, en Ecuador, el crecimiento de la renta petrolera se explica por un cambio en el marco regulatorio orientado hacia este fin, además de un crecimiento en la participación de las empresas públicas.

\section{- Participación de las empresas públicas}

A lo largo de este estudio se ha destacado la importancia de la participación de las empresas públicas; el reflejo del aporte que hacen en términos productivos, también se puede observar en los ingresos que "captan" para sus respectivos Estados. Ahora bien, aunque hay diferencias en la naturaleza jurídica de estas empresas, la

16. Solamente se toma estas fuentes puesto que hace posible la comparación entre los dos países. Además, son las más significativas. 
importancia que adquieren es trascendental para entender la política recaudatoria de los dos países.

Cuadro 7. Composición de los ingresos Sector Público Nivel Central (\% del PIB).

\begin{tabular}{|c|c|c|c|c|c|c|c|c|c|c|c|}
\hline \multirow{3}{*}{ Años } & \multicolumn{5}{|c|}{ ECUADOR } & \multicolumn{6}{|c|}{ COLOMBIA } \\
\hline & \multirow{2}{*}{$\begin{array}{l}\text { DIRECTOS } \\
\text { (RENTA) }\end{array}$} & \multirow{2}{*}{$\begin{array}{l}\text { INDIRECTOS } \\
\text { (IVA) }\end{array}$} & \multirow{2}{*}{ PETROLEROS } & \multirow{2}{*}{$\begin{array}{c}\text { TOTAL } \\
\text { INGRESOS }\end{array}$} & \multirow{2}{*}{$\begin{array}{c}\text { INGREESOS } \\
\text { PETROLEROS } \\
\text { COMO \% DE } \\
\text { INGRESOS } \\
\text { GOBIERNO } \\
\end{array}$} & \multicolumn{2}{|c|}{ DIRECTOS (RENTA) } & \multirow{2}{*}{$\begin{array}{c}\text { INDIRECTOS } \\
\text { (IVA) }\end{array}$} & \multirow{2}{*}{ PETROLEROS } & \multirow{2}{*}{$\begin{array}{c}\text { TOTAL } \\
\text { INGRESOS }\end{array}$} & \multirow{2}{*}{\begin{tabular}{|c|} 
INGRESSOS \\
PETROLEROS \\
COMO \% DE \\
INGRESOS \\
GOBIERNO \\
\end{tabular}} \\
\hline & & & & & & Total & Sin petróleo & & & & \\
\hline 2002 & 1,9 & 6,1 & 4,8 & 16,0 & 29,8 & 4,1 & 3,6 & 4,3 & 1,0 & 12,3 & 8,3 \\
\hline 2003 & 1,8 & 5,4 & 4,8 & 14,7 & 32,7 & 4,3 & 3,8 & 4,8 & 0,9 & 12,6 & 7,2 \\
\hline 2004 & 1,9 & 5,3 & 4,3 & 14,2 & 30,1 & 4,9 & 4,2 & 4,9 & 1,1 & 12,9 & 8,4 \\
\hline 2005 & 2,3 & 5,3 & 3,8 & 14,6 & 25,9 & 5,1 & 4,4 & 5,1 & 1,1 & 13,5 & 8,1 \\
\hline 2006 & 2,3 & 5,3 & 3,7 & 14,7 & 24,9 & 5,5 & 4,6 & 5,6 & 1,4 & 14,7 & 9,8 \\
\hline 2007 & 2,5 & 5,3 & 3,5 & 16,6 & 20,8 & 5,6 & 4,7 & 5,4 & 1,6 & 15,0 & 10,9 \\
\hline 2008 & 3,8 & 5,3 & 7,5 & 22,3 & 33,6 & 5,1 & 4,2 & 5,6 & 1,7 & 15,6 & 11,0 \\
\hline 2009 & 4,0 & 5,5 & 3,7 & 18,5 & 19,8 & 5,6 & 4,3 & 5,1 & 2,9 & 15,3 & 18,8 \\
\hline 2010 & 3,5 & 6,5 & 6,5 & 22,2 & 29,3 & 4,8 & 4,2 & 5,3 & 1,3 & 13,8 & 9,1 \\
\hline 2011 & 3,9 & 6,2 & 7,7 & 22,1 & 34,7 & 5,4 & 4,5 & 5,6 & 1,7 & 15,2 & 11,3 \\
\hline 2012 & 3,9 & 7,2 & 7,2 & 23,1 & 31,2 & 6,6 & 4,9 & 5,5 & 2,8 & 16,1 & 17,1 \\
\hline
\end{tabular}

Fuente: elaboración propia con información del Banco Central del Ecuador, Ministerio de Hacienda - Colombia y Asociación Colombiana de Petróleos (ACP)

Ecopetrol S.A. es la empresa más importante de Colombia, entre otras cosas, por el monto de ingresos que le genera al Estado. Los aportes al Gobierno Nacional Central (GNC) se concentran en dos fuentes principales: renta y complementarios, y dividendos. Pero además, hay una participación que no deja de ser significativa, que son las regalías. Si se tiene en cuenta esta fuente, la importancia del petróleo en la estructura fiscal se hace mucho mayor.

Durante el periodo 2008 - 2012, Ecopetrol S.A., aportó, en promedio, el 15\% de los ingresos de la nación, lo que representa algo más del 3\% del PIB (ver Cuadro 8). En el caso del impuesto a la renta, la contribución fue del 13\%. Si se toma como referencia el año 2012 la participación fue del 23\%. Esta información ratifica la importancia que tiene esta empresa para el Estado, particularmente desde el punto de vista fiscal.

Sin embargo, para la empresa, el hecho de que tenga que pagar dividendos le genera menos posibilidades para afianzarse dentro de su estrategia de expansión. No obstante, por lo que se puede observar, hay un margen de recursos que le quedan para desarrollar sus labores operativas y de inversión. Prueba de esto es el crecimiento de las utilidades generadas durante los últimos años. Se podría entender que el periodo de bonanza se está aprovechando para fortalecer la empresa, logrando consolidarse como una de las más importantes del sector en $\mathrm{AL}^{17}$.

En el caso de Ecuador, no se puede hacer el mismo tipo de análisis que en Colombia, puesto que, como se mencionó, las empresas tienen una naturaleza jurídica distinta. EP Petroecuador es una empresa pública, creada mediante Decreto Ejecutivo 315 del 6 de abril de 2010, que desarrolla las modificaciones introducidas en la Ley de Hidrocarburos y, de Empresas Públicas (LOEP ${ }^{\mathbf{1 8}}$ ). La forma como se hacen las "transferencias" al Estado es distinta respecto a como lo hace Ecopetrol.

17. Para el año 2012, Ecopetrol se ubicó dentro de las 40 empresas petroleras más grandes del mundo, y la cuarta en el continente americano (Ecopetrol, 2013: 17)

18. La Ley Orgánica de Empresas Públicas (LOEP) fue promulgada en el año 2009. 


\section{Cuadro 8. Ecopetrol S.A. - Impuestos, regalías y transferencias realizadas al gobierno central y descentralizados.}

\begin{tabular}{|c|c|c|c|c|c|c|c|c|}
\hline Año & $\begin{array}{c}\text { Impuesto a la } \\
\text { renta y } \\
\text { dividendos } \\
\text { (billones de } \\
\text { pesos } \\
\text { colombianos) }\end{array}$ & $\begin{array}{c}\text { Regalías } \\
\text { (billones de } \\
\text { pesos } \\
\text { colombianos) }\end{array}$ & $\begin{array}{c}\text { Otros } \\
\text { (billones de } \\
\text { pesos } \\
\text { colombianos) }\end{array}$ & $\begin{array}{c}\text { Total } \\
\text { (billones de } \\
\text { pesos } \\
\text { colombianos) }\end{array}$ & \% PIB & $\begin{array}{c}\text { \% ingresos } \\
\text { corrientes } \\
\text { Gobierno } \\
\text { Central }\end{array}$ & $\begin{array}{c}\text { Utilidades } \\
\text { (billones de } \\
\text { pes os } \\
\text { colombianos) }\end{array}$ & $\begin{array}{c}\text { dividendos } \\
\text { pagados al } \\
\text { gobie rno/ } \\
\text { utilidades } \\
\text { (\%) }\end{array}$ \\
\hline 2002 & 2,08 & 1,78 & 1,61 & 5,48 & 2,2 & 13,5 & 1,33 & \\
\hline 2003 & 1,78 & 2,26 & 1,65 & 5,69 & 2,1 & 10,8 & 1,58 & 81,3 \\
\hline 2004 & 2,20 & 2,37 & 1,81 & 6,38 & 2,1 & 10,9 & 2,11 & 73,3 \\
\hline 2005 & 2,45 & 1,90 & 2,03 & 6,37 & 1,9 & 10,5 & 3,25 & 61,5 \\
\hline 2006 & 3,67 & 3,68 & 2,22 & 9,57 & 2,5 & 11,4 & 3,39 & 61,5 \\
\hline 2007 & 6,39 & 3,91 & 2,15 & 12,45 & 2,9 & 14,7 & 5,18 & 132,0 \\
\hline 2008 & 6,80 & 5,60 & 1,95 & 14,35 & 3,0 & 13,5 & 11,60 & 81,1 \\
\hline 2009 & 12,61 & 4,36 & 1,70 & 18,66 & 3,7 & 21,8 & 6,20 & 69,0 \\
\hline 2010 & 4,70 & 5,34 & 1,73 & 11,77 & 2,2 & 9,5 & 8,30 & 63,7 \\
\hline 2011 & 9,40 & 8,05 & 1,94 & 19,39 & 3,1 & 13,4 & 15,40 & 63,6 \\
\hline 2012 & 17,14 & 8,56 & 1,58 & 27,28 & 4,1 & 19,4 & 15,00 & 43,0 \\
\hline
\end{tabular}

Fuente: elaboración propia con datos de Ecopetrol S.A y Ministerio de Hacienda

* Las utilidades generadas en un ejercicio se distribuyen para el siguiente, por tanto, el cálculo guarda correspondencia con el procedimiento contable. También se aclara que además de pagarle dividendos al Estado, también se entregan al resto de accionistas minoritarios (a partir del ejercicio 2009).

Además, a pesar de que EP Petroecuador, goza de autonomía presupuestaria, financiera, económica, administrativa y de gestión, es el Banco Central quien recibe y distribuye los ingresos generados fruto de la comercialización interna y externa tanto del crudo como de derivados (Campodónico, 2008). Las empresas reciben inicialmente el monto de los costos y gastos en los que han incurrido, y presentan su plan de inversión. Los excedentes, se transfieren directamente al Presupuesto General del Estado (PGE).

En el Cuadro 9 se muestra que de las utilidades generadas por EP Petroecuador, el porcentaje trasladado al gobierno, es muy elevado. El excedente que queda para reinversiones es muy pequeño, excepto el último año. Comparado con el caso colombiano, los dividendos que paga Ecopetrol al Estado, representan una proporción mucho menor de los beneficios. Este factor va a redundar en la política de fortalecimiento de este tipo de empresas.

En cualquier caso, las empresas públicas de los dos países se convierten en un instrumento fundamental para el recaudo de ingresos a favor del Estado. Se debe resaltar este aspecto en el caso colombiano, dado que, este objetivo se alcanza, por la presencia de Ecopetrol, y no tanto, por la entrada de empresas extranjeras. De esta manera, su modelo de explotación petrolera, aun con las diferencias respecto a Ecuador, logra capturar algunos de los aspectos señalados para el neoextractivismo. Este hecho ratifica la visión que se había dado sobre los alcances y limitaciones del marco regulatorio. 


\section{Cuadro 9. EP Petroecuador. Utilidades y transferencias al Presupuesto General del Estado (Cifras en miles de dólares)}

\begin{tabular}{|c|c|c|c|c|c|c|}
\hline \multirow[b]{2}{*}{ Año } & \multirow[b]{2}{*}{ Utilidad Neta } & \multicolumn{5}{|c|}{ Transferencias al Estado } \\
\hline & & Total & $\begin{array}{c}\text { Presupuesto } \\
\text { General del } \\
\text { Estado }\end{array}$ & $\begin{array}{c}\text { Partícipes } \\
* *\end{array}$ & \begin{tabular}{|c|}
$\%$ de las utilidades \\
transferidas al \\
Presupuesto General \\
del Estado
\end{tabular} & $\begin{array}{c}\% \text { transferencias } \\
\text { totales }\end{array}$ \\
\hline 2004 & 2.041 .418 & 2.130 .862 & 1.202 .240 & 928.623 & 58,9 & 104,4 \\
\hline 2005 & 2.300 .806 & 2.315 .420 & 1.190 .000 & 1.125 .000 & 51,7 & 100,6 \\
\hline 2006 & 3.219 .400 & 3.183 .511 & 1.817 .041 & 1.366 .470 & 56,4 & 98,9 \\
\hline 2007 & 2.969 .531 & 3.086 .813 & 1.402 .253 & 1.684 .560 & 47,2 & 103,9 \\
\hline 2008 & 5.072 .366 & 4.666 .302 & 3.905 .734 & 760.569 & 77,0 & 92,0 \\
\hline 2009 & 3.276 .437 & 2.927 .374 & 2.927 .372 & 2 & 89,3 & 89,3 \\
\hline 2010 & 3.514 .445 & 2.810 .163 & 2.810 .163 & N.A. & 80,0 & 80,0 \\
\hline 2011 & 6.260 .328 & 6.226 .443 & 6.226 .443 & N.A. & 99,5 & 99,5 \\
\hline 2012 & 5.864 .482 & 4.832 .379 & 4.832 .379 & N.A. & 82,4 & 82,4 \\
\hline
\end{tabular}

* Los datos para el año 2010 corresponden al periodo comprendido entre el 4 de abril al 31 de diciembre de 2010. Cambio de naturaleza jurídica de EP Petroecuador.

** Aquí se contabilizan las transferencias que corresponden a distintos pagos y deducciones tales como: Fondo de Ecodesarrollo, Junta de Defensa, Regalías etc. Hasta 2008, también se incluían los costos de importación de derivados. De ahí en adelante, se vienen realizando modificaciones en el marco de los cambios introducidos en la Ley de Hidrocarburos.

\section{- Participación del Estado en la renta petrolera}

La renta petrolera se define como la diferencia entre el precio internacional del recurso natural (petróleo) y los costos de producción (Campodónico, 2008). La participación del Estado en la misma depende de varios factores, tales como: las medidas de carácter impositivo y en general, regulatorio y, la importancia que adquieren las empresas públicas. El cálculo que se presenta en este estudio tiene algunas limitaciones puesto que en ninguno de los dos países se puede separar la explotación y comercialización de crudo y derivados. Estas últimas actividades no formarían parte de la renta petrolera apropiada por el Estado (Ibíd.).

El Cuadro 10 muestra que la capacidad del Estado para apropiarse de los excedentes generados en la actividad petrolera, ha crecido en los dos países; empero, los cambios más importantes se presentan en Ecuador. Mientras la proporción de la renta con que se queda el Estado venía disminuyendo hasta 2005, hay un aumento considerable en los años siguientes, logrando que, a 2011, prácticamente se haya duplicado esta relación. En el caso de Colombia es más estable, logrando ubicarse en un porcentaje de alrededor del 35\%. De esta manera, entre tanto en Ecuador se pueden observar cambios que muestran que hay una estrategia deliberada (modificación marco regulatorio) para incrementar los ingresos fiscales, en el caso de Colombia, no se vislumbran cambios sustanciales. Cabe recordar que en la revisión de literatura se destacaba este aspecto como un paso fundamental en aquellos países con abundancia de un recurso como lo es el petróleo. 
Cuadro 10. Participación del Estado en la renta petrolera (\% del PIB)

\begin{tabular}{|c|c|c|c|c|c|c|}
\hline \multirow{2}{*}{ AÑO } & \multicolumn{3}{|c|}{ COLOMBIA } & \multicolumn{3}{c|}{ ECUADOR } \\
\cline { 2 - 7 } & RENTA & $\begin{array}{c}\text { INGRESOS } \\
\text { FISCALES } \\
\text { PETROLOLEROS }\end{array}$ & $\begin{array}{c}\text { \% ESTADO } \\
\text { RENTA } \\
\text { PETROLERA }\end{array}$ & $\begin{array}{c}\text { RENTA } \\
\text { PETROLERA }\end{array}$ & $\begin{array}{c}\text { INGRESOS } \\
\text { FISCALES } \\
\text { PETROLEROS }\end{array}$ & $\begin{array}{c}\text { \% ESTADO } \\
\text { RENTA } \\
\text { PETROLERA* }\end{array}$ \\
\hline 2003 & 4,3 & 1,0 & 23,8 & 11,7 & 4,9 & 41,9 \\
\hline 2004 & 5,0 & 0,9 & 18,2 & 13,0 & 5,1 & 39,5 \\
\hline 2005 & 5,4 & 1,9 & 35,9 & 19,4 & 5,8 & 29,8 \\
\hline 2006 & 6,3 & 1,9 & 31,2 & 25,4 & 5,3 & 21,0 \\
\hline 2007 & 6,9 & 2,4 & 35,1 & 27,4 & 7,4 & 26,8 \\
\hline 2008 & 5,9 & 2,5 & 41,5 & 26,4 & 7,5 & 28,3 \\
\hline 2009 & 7,7 & 2,9 & 37,5 & 30,2 & 14,5 & 47,9 \\
\hline 2010 & 5,2 & 3,7 & 70,6 & 17,2 & 8,1 & 47,0 \\
\hline 2011 & 6,6 & 2,4 & 35,7 & 20,4 & 11,3 & 55,6 \\
\hline 2012 & 8,8 & 3,1 & 35,4 & 25,6 & 13,6 & 53,2 \\
\hline
\end{tabular}

Fuente: elaboración propia a partir de información del Banco Central del Ecuador, Ministerio de Hacienda (Colombia), Banco Mundial y el Informe Estadístico Petrolero (IEP)-ACP de 2014.

* En el caso de Ecuador, se toma como referencia el Gobierno Nacional Consolidado.

\section{Uso de la renta petrolera}

El objetivo del análisis del uso de la renta petrolera es identificar si existe algún patrón que denote la manera como son utilizados los ingresos que obtiene el Estado fruto de su participación en la renta petrolera. Como ya se anotó en la revisión del marco regulatorio, no es fácil determinar el uso que se da a este tipo de recursos por dos razones: en primer lugar, porque hay una parte de los ingresos petroleros que quedan incluidos en presupuesto general del Estado, sin haber una señalización frente a uso, con la cual, no se podría discriminar la forma como se financian las actividades de gasto; y en segundo lugar, los ingresos que son transferidos hacia entes subnacionales, como en el caso de las regalías, pueden quedar dispersos en un sinnúmero de proyectos donde no se podría establecer un marco común de análisis. No obstante, además de las transferencias al nivel central, donde no hay una destinación específica, se podría hacer referencia a dos clases de usos: subsidios a los combustibles y constitución de fondos (Portillo, 2014).

\section{- Subsidios a los combustibles}

En el caso de Ecuador, la Ley de Hidrocarburos otorga al Presidente de la República la potestad para regular los precios de comercialización de los derivados de petróleo. Dadas estas facultades, que no son nuevas, pero que tampoco se han modificado, se ha mantenido una política de subsidios que representan un elevado costo fiscal. El financiamiento de este tipo de gastos se realiza a través de los excedentes generados en la balanza comercial de derivados petroleros y los demás ingresos fruto de la operación de empresas públicas. 
El cuadro 11 muestra el desequilibrio que genera para Ecuador la importación de derivados. Lo que se observa es que hay un creciente desajuste, lo cual confirma el impacto que dicha situación ocasiona en las finanzas del Estado. Este monto al final se computa como una reducción de las transferencias que le hace EP Petroecuador al Gobierno Central. Así las cosas, uno de los usos de la renta petrolera es precisamente la financiación de este tipo de gastos, que de ninguna manera contribuyen a su estrategia de desarrollo. Dicha situación también sería contradictoria con la idea de buscar mayor eficiencia del Estado, tal y como se insiste bajo el neoextractivismo.

\section{Cuadro 11. Ecuador - subsidios a las importaciones de derivados}

\begin{tabular}{|c|c|c|c|c|c|c|c|c|c|}
\hline Concepto & 2004 & 2005 & 2006 & 2007 & 2008 & 2009 & 2010 & 2011 & 2012 \\
\hline $\begin{array}{l}\text { Costos totales } \\
\text { importaciones } \\
\text { (millones de } \\
\text { dólares) }\end{array}$ & 829 & 1.474 & 1.952 & 2.435 & 2.878 & 2.239 & 3.586 & 4.408 & 5.009 \\
\hline \begin{tabular}{|l|} 
Ingresos totales \\
ventas internas \\
(millones de \\
dólares) \\
\end{tabular} & 554 & 745 & 878 & 1.008 & 987 & 1.154 & 1.569 & 1.458 & 1.604 \\
\hline \begin{tabular}{|l|} 
Diferencia de \\
ingresos y costos \\
(millones de \\
dólares)
\end{tabular} & (275) & (729) & (1.074) & (1.427) & (1.891) & (1.085) & (2.017) & (2.950) & (3.405) \\
\hline
\end{tabular}

Fuente: elaboración propia con información del Banco Central del Ecuador.

En el caso de Colombia el manejo de los subsidios a los combustibles dista mucho del planteado para Ecuador. En primer lugar, Ecopetrol no es parte del Sector Público No Financiero (SPNF), por lo cual, su operación es independiente de la del gobierno, y por tanto, no hay lugar a subsidios implícitos (Rincón et al., 2008). De esta manera, es el GNC quien inicialmente asumió este gasto, que posteriormente fue trasladado al Fondo de Estabilización de los Precios de los Combustibles (FEPC). En segundo lugar, a pesar de que los precios de los combustibles también los fija el gobierno nacional, la referencia que se toma para el cálculo del subsidio es el precio de paridad internacional. Así las cosas, no hay un uso directo de la renta petrolera asociado al consumo de los combustibles. Es más, según Rincón et al. (2008) dado que el GNC y los entes subnacionales recaudan impuestos por concepto de la comercialización de los combustibles, estos ingresos superarían el monto de subsidios computados, razón por la cual, no habría una subvención neta hacia el consumidor.

\section{Constitución de fondos}

La idea de constitución de fondos ha sido ampliamente difundida dentro de la literatura como un mecanismo para estabilizar los ingresos de bienes cuyos precios son fluctuantes, como es el caso de las materias primas, entre ellas, el petróleo. Lo que se busca es que haya un instrumento que suavice los ciclos económicos y particularmente, las finanzas públicas. 
En el caso ecuatoriano, dada la importancia de los ingresos petroleros, se habían constituido varios fondos y preasignaciones durante la primera parte de la década de los 2000. Los fondos creados fueron los siguientes: Fondo de Estabilización Petrolera (FEP), Fondo de Estabilización, Inversión Social y Productiva y Reducción del Endeudamiento Público (FEIREP), Cuenta Especial de Reactivación Productiva y Social, del Desarrollo Científico Tecnológico y de la Estabilización Fiscal (CEREPS) que reemplazó al FEIREP, Fondo de Ahorros y Contingencias (FAC) y, Fondo Ecuatoriano de Inversión en Sectores Estratégicos e Hidrocarburíferos (FEISEH). En el año 2008, bajo el argumento de que estos fondos se habían utilizado excesivamente para financiar el pago de la deuda y compensar las variaciones de los ingresos petroleros, en detrimento de la inversión social, se decidió eliminarlos. La única preasignación que se mantuvo fue la del Fondo para el Ecodesarrollo Regional Amazónico (ECORARE) ${ }^{\mathbf{1 9}}$. Los excedentes fueron trasladados al Presupuesto General del Estado (Cueva y Ortiz, 2013).

En el caso de Colombia, dejando de lado los fondos constituidos en el marco de la administración del nuevo Sistema Nacional de Regalías ${ }^{\mathbf{2 0}}$, no existe ningún otro esfuerzo que se enmarque en la idea de la constitución de un tipo de instrumento de esta naturaleza. Lo único que quedaría por destacar son los recursos destinados al financiamiento del Fondo Nacional de Pensiones Territoriales (FONPET), y la constitución de un patrimonio autónomo para atender el pasivo pensional de Ecopetrol (Rincón et al., 2008).

Bajo las consideraciones planteadas anteriormente, la revisión del uso de la renta petrolera queda muy limitada, en tanto, no existe información suficiente que permita conocer el destino que se le está dando. Tampoco se podría decir que en el caso ecuatoriano, se está destinando, necesariamente, al desarrollo de programas sociales, tal y como se advertía en la revisión de literatura. No obstante, se entiende que el hecho de que se tengan recursos para inversión desde el sector petrolero, permite liberar fondos de otras fuentes de financiamiento. De cualquier manera, el monto asignado para subsidios a los combustibles va en contra de la idea de estructurar un nuevo modelo de explotación petrolera. Por otro lado, se puede vislumbrar que las modificaciones en el marco regulatorio son mucho más enfáticas en el caso del recaudo que en el del gasto, lo cual plantea limitantes.

Los elementos más resaltables de esta dimensión son los siguientes: en primer lugar, en los dos países hay un crecimiento notable de los ingresos petroleros, donde la participación de las empresas públicas resulta fundamental; en segundo lugar; se observó que en el caso Ecuatoriano, el Estado logra captar una mayor proporción de la renta petrolera, que en parte se explica por las modificaciones introducidas en el marco regulatorio; en tercer lugar, siendo una limitante importante en los dos casos, no se observan instrumentos concretos para el uso de la renta petrolera; y por último, para el caso de Ecuador, el financiamiento de subsidios a los combustibles con los ingresos petroleros, resulta, a todas luces, un mecanismo muy regresivo e ineficiente. Los elementos descritos se identifican claramente con los

19. Creado mediante la Ley 10 de 1992 y luego modificado en el año 2008.

20. Ver Acto Legislativo No. 05 de 2011. 
planteamientos neoextractivistas, pero visto desde el lado de los ingresos, no así con los gastos donde no se puede establecer una estrategia deliberada para su uso.

\section{CONCLUSIONES}

Uno de los objetivos propuestos dentro de esta investigación fue, aportar información empírica que permitiera diferenciar las dos variantes del extractivismo señaladas por Gudynas (2011a, 2011b, 2012). Con este fin, se hizo la comparación de las principales características de los modelos de explotación petrolera de los países seleccionados.

Por los aportes derivados de la revisión de literatura y el análisis los modelos de explotación petrolera, la diferencia fundamental viene dada por el rol que asume el Estado. Los cambios introducidos en este sentido fueron observados para el caso ecuatoriano. Los rasgos más importantes son: en primer lugar, el Estado busca asumir un mayor control de la actividad petrolera, lo cual fue posible gracias a las modificaciones introducidas en el marco regulatorio y al papel activo que se le otorga a las empresas públicas; en segundo lugar, fruto de lo anterior, hay un cambio trascendental de las relaciones con las empresas transnacionales; y en tercer lugar, como resultado de una mayor participación estatal en la producción petrolera y el incremento en la presión fiscal, el Estado fue capaz de desarrollar una política activa para capturar una mayor parte de la renta petrolera. En el caso ecuatoriano dicha participación pasa del 29\% en 2005, a 53\% en 2012. En Colombia, esta tasa permanece en una media del $35 \%$. Los elementos mencionados guardan correspondencia con los aportes citados bajo el neoextractivismo.

Las cifras encontradas también muestran dinámicas divergentes en los dos países, las cuales se explican, en buena medida, por las características de sus modelos de explotación petrolera. En cuanto a la producción de crudo, en el caso de Colombia, creció a una tasa media anual del 12. Por el contrario, en Ecuador, mientras en el periodo 2000 - 2006 la producción venía creciendo, a partir de 2007, empieza a descender, y permanece estancada hasta el 2012. A pesar de este fenómeno, en los dos países, las exportaciones petroleras resultan significativas; en el caso ecuatoriano, representan más del 50\% del total, y en Colombia, a 2012, este porcentaje llega a ser del 44\%, cuando en el año 2004, representaban el 17\%.

No obstante, hay elementos básicos que sí es importante resaltar. En el caso colombiano, la participación de empresas públicas no se ha modificado sustancialmente, aunque permanece alta. En Ecuador, sucede lo contrario. Mientras en el año 2006 las empresas estatales producían el 46\% del petróleo, en 2012, dicha participación pasó a ser del 73\%.

Por otro lado, a pesar de los cambios introducidos en el marco regulatorio, los avances de Ecuador en el proceso de industrialización del petróleo, que serían parte de esa estrategia neoextractivista, no se puede decir que sean satisfactorios. El estancamiento de la producción de derivados, paralelo al aumento de consumo interno, generan desajustes en su balanza comercial. Esto se presenta dado que el consumo de derivados, en general, se incrementa de manera muy notable, superando la producción desde 2005. La participación de los productos derivados en el total de importaciones tiende a profundizarse, representado, entre 2006 y 2012, como 
media, el 20\%. Si a esto se le añade la baja dinámica en la producción de crudo, las barreras o limitantes del modelo petrolero ecuatoriano se hacen más visibles.

Lo más preocupante para Ecuador es el deterioro del saldo en la balanza comercial de derivados, fenómeno que se profundiza a partir de 2005, pero continúa con un ritmo acelerado bajo el gobierno de Rafael Correa. En el año 2012, el déficit llega a representar el 5.2\% del PIB, algo que va a tener repercusiones directas en el presupuesto del gobierno. En el caso de Colombia, a pesar que en casi todos los años tiene un superávit, en 2012, el saldo pasa a ser deficitario.

Para entender las diferentes dinámicas de producción petrolera, el papel que juega la IED resulta fundamental. En el caso colombiano, las medidas adoptadas para atraer IED efectivamente han funcionado. En el periodo 2007 - 2012, en Ecuador, la IED realizada en el sector petrolero en Ecuador fue de US\$ 929 millones, mientras en Colombia el monto ascendió a US\$ 22.402 millones. De ahí que el rol que asume la IED en los dos países genera una diferencia importante en la trayectoria productiva durante los últimos años.

En Colombia, a partir de los cambios realizados en el marco regulatorio y un contexto externo favorable, el objetivo de incrementar la producción, se ha logrado, no obstante, las reservas presentan niveles muy bajos, y la amenaza de desabastecimiento está presente. Ahora bien, los efectos positivos se pueden visualizar en el caso del crudo, no así en los derivados. Las importaciones se incrementaron a un ritmo muy alto, particularmente en los últimos tres años. Esto hace que al finalizar el 2012, la participación de los combustibles y lubricantes en el total de importaciones llegue a representar el 9.7\%. De esta manera, Colombia deja de ser un exportador neto, ratificando su condición extractivista.

Como un elemento destacable, se resalta el papel que asume una empresa como Ecopetrol S.A., quien, por lo que se pudo ver, participa activamente a lo largo de toda la cadena del petróleo ${ }^{\mathbf{2 1}}$, además de aportar la mayor parte de la renta petrolera que capta el Estado colombiano. Dichas singularidades configuran un modelo, que aunque difiere del caso ecuatoriano, tampoco se puede decir que se pueda amoldar estrictamente a los rasgos citados bajo el extractivismo clásico.

Por último, haciendo la comparación de los referentes conceptuales en torno a los dos tipos de extractivismos y la evolución de la dinámica productiva y fiscal, las diferencias ya no son tan evidentes.

21. Excepto en la distribución. 


\section{REFERENCIAS}

1. CAMPODÓNICO, Humberto (2004). Reformas e inversión en la industria de hidrocarburos de América Latina. Revista de la CEPAL, serie "Recursos Naturales e Infraestructura", núm. 78, Santiago de Chile.

2. (2007). La gestión de la industria de hidrocarburos con predominio de empresas del Estado. CEPAL/GTZ, serie “Recursos Naturales e Infraestructura”, núm. 121, Santiago de Chile.

3. (2008). Renta petrolera y minera en países seleccionados de América Latina, CEPAL/GTZ, Documento de proyecto, Santiago de Chile.

4. CUEVA, Simón y ORTIZ, María (2013). Ingresos fiscales por explotación de hidrocarburos en Ecuador. Departamento de países del grupo andino, Título IV, Serie IDB-PB-198, Banco Interamericano de Desarrollo.

5. GUDYNAS, Eduardo (2011a). Más allá del nuevo extractivismo: transiciones sostenibles y alternativas al desarrollo. En: el desarrollo en cuestión, reflexiones desde América Latina, Fernanda Wanderley (Coord.), Oxfam y CIDES UMSA, Bolivia, pp. 379-410.

6. (2011b). "Alcances y contenidos de las transiciones al Post-Extractivismo", En: Ecuador Debate, núm. 82, Quito, pp. 61-80.

7. (2012). “Estado compensador y nuevos extractivismos las ambivalencias del progresismo sudamericano". En: Revista Nueva Sociedad, núm. 237, pp. 128-146.

8. (2013). "Extracciones, extractivismos y extrahecciones. Un marco conceptual sobre la apropiación de los recursos naturales". Observatorio del desarrollo No. 18. Centro Latinoamericano de Ecología Social.

9. PÉREZ, Carlota (2010). “Dinamismo tecnológico e inclusión social en América Latina: una estrategia de desarrollo productivo basada en los recursos naturales". Revista de la CEPAL, núm. 100, pp. 123-145, Santiago de Chile.

10. PORTILLO RIASCOS, Luis Hernando (2014). "Extractivismo clásico y neoextractivismo, ¿dos tipos de extractivismos diferentes? (primera parte)". Revista Tendencias, Vol. XV, No. 2, 2do. semestre de 2014, Facultad de Ciencias Económicas y Administrativas, Universidad de Nariño, pp. 11-29.

11. RAMOS, Joseph (1999). "Una estrategia de desarrollo a partir de los complejos productivos (clusters) en torno a los recursos naturales ¿una estrategia prometedora?". Revista de la CEPAL, núm. 66, pp. 105-125, Santiago de Chile.

12. RINCÓN, Hernán; LOZANO, Ignacio y RAMOS, Jorge. (2008).“Rentas petroleras, subsidios e impuestos a los combustibles en Colombia: ¿qué ocurrió durante el reciente choque de precios?". Borradores de Economía, núm. 541, Banco de la República.

13. UNIDAD DE PLANEACIÓN MINERO ENERGÉTICA [UPME] (2013). Cadena del Petróleo 2013. Bogotá. 